\title{
The Fornax Deep Survey with the VST
}

\section{A size and magnitude limited catalog of dwarf galaxies in the area of the Fornax cluster (Corrigendum)}

\author{
Aku Venhola ${ }^{1,2}$, Reynier Peletier ${ }^{2}$, Eija Laurikainen ${ }^{1}$, Heikki Salo $^{1}$, Enrichetta Iodice ${ }^{3}$, Steffen Mieske ${ }^{4}$, \\ Michael Hilker ${ }^{5}$, Carolin Wittmann ${ }^{6}$, Thorsten Lisker ${ }^{6}$, Maurizio Paolillo ${ }^{3,7}$, Michele Cantiello ${ }^{8}$, Joachim Janz ${ }^{1,15}$, \\ Marilena Spavone ${ }^{3}$, Raffaele D'Abrusco ${ }^{9}$, Glennvande Ven ${ }^{5,10}$, Nicola Napolitano ${ }^{3}$, Gijs Verdoes Kleijn ${ }^{2}$, \\ Natasha Maddox ${ }^{12}$, Massimo Capaccioli ${ }^{11}$, Aniello Grado ${ }^{3}$, Edwin Valentijn ${ }^{2}$, \\ Jesús Falcón-Barroso ${ }^{13,14}$, and Luca Limatola ${ }^{3}$ \\ 1 Astronomy Research Unit, University of Oulu, Finland \\ e-mail: avenhola@univ.yo.oulu.fi \\ 2 Kapteyn Institute, University of Groningen, Groningen, The Netherlands \\ 3 INAF - Astronomical Observatory of Capodimonte, Salita Moiariello 16, 80131 Naples, Italy \\ ${ }^{4}$ European Southern Observatory, Alonso de Cordova 3107, Vitacura, Santiago, Chile \\ 5 European Southern Observatory, Karl-Schwarzschild-Strasse 2, 85748 Garching bei München, Germany \\ ${ }^{6}$ Astronomisches Rechen-Institut, Zentrum für Astronomie der Universität Heidelberg, Mönchhofstraße 12-14, 69120 Heidelberg, \\ Germany \\ 7 University of Naples Federico II, C.U. Monte Sant'Angelo, Via Cinthia, 80126 Naples, Italy \\ 8 INAF Osservatorio Astronomico di Teramo, Via Maggini, 64100 Teramo, Italy \\ 9 Smithsonian Astrophysical Observatory, 60 Garden Street, 02138 Cambridge, MA, USA \\ ${ }^{10}$ Max-Planck-Institut für Astronomie, Königstuhl 17, 69117 Heidelberg, Germany \\ 11 University of Naples Federico II, C.U. Monte Sant'Angelo, Via Cinthia, 80126 Naples, Italy \\ 12 ASTRON, The Netherlands Institute for Radio Astronomy, Postbus 2, 7990 AA Dwingeloo, The Netherlands \\ 13 Instituto de Astrofisica de Canarias, C/ Via L'actea s/n, 38200 La Laguna, Spain \\ 14 Depto. Astrofisica, Universidad de La Laguna, C/ Via L'actea s/n, 38200 La Laguna, Spain \\ 15 Finnish Centre of Astronomy with ESO (FINCA), University of Turku, Väisäläntie 20, 21500 Piikkiö, Finland
}

A\&A 620, A165 (2018) https://doi .org/10.1051/0004-6361/201833933

Key words. galaxies: clusters: individual: Fornax - galaxies: dwarf - galaxies: photometry - galaxies: structure - errata, addenda

An error occurred during the production process of the original article pertaining to the numbering of the figures beginning with Fig. 18. Figure 20 is incorrectly labeled and it should be named
Fig. 18, and so on. However, the citations of the figures in the text are consistent with their respective image. 